\title{
Paysage suisse et imaginaire minéral chez Henry Bauchau
}

Les journaux d'Henry Bauchau, on le sait, sont un élément clé dans la réception de son œuvre; au-delà de nombreux renseignements sur le processus créateur, ces écrits ont le mérite de fournir des informations importantes sur les sources qui ont irrigué son imaginaire. Ainsi, le lecteur désireux de repérer les éléments sous-jacents à cet imaginaire a la chance d'y trouver des remarques précises sur les expériences qui ont engendré l'écriture des textes et la production des images chers à cet auteur.

En travaillant à cerner l'imaginaire du minéral chez Henry Bauchau, nous avons relevé un certain nombre de notes qui relient l'expérience de l'espace minéral des Alpes bernoises à des constructions et à des images littéraires. Selon le témoignage du poète, le recueil Géologie est né de la contemplation des montagnes:

Quand j'écris Géologie, j'habite Gstaad depuis cinq ans. La contemplation quotidienne des montagnes, la succession des saisons dans le même paysage, l'écoute constante du torrent qui s'écoule apaisé ou tumultueux sous notre chalet y font sentir leur présence. C'est elle qui me conduit de page en page et de pensées en méditations à une sorte de conclusion ou décision abrupte sur laquelle le poème se clôt. ${ }^{201}$

En effet, les motifs de la montagne et du torrent structurent ce recueil et déclenchent des méditations sur la construction de soi comme de l'œuvre, sur le langage et le temps. De même, La Chine intérieure "est née d'une inspiration soudaine, un matin de janvier à la montagne» ${ }^{\mathbf{2 0 2}}$ et certaines images sont tributaires du séjour à Gstaad: "Les mots neige, montagne et plusieurs autres ont certainement pris une signification singulière en moi à la suite de mon long séjour - vingt-quatre ans - dans la montagne suisse „203. De même encore, l'écoute de ce paysage qui produit des sensations denses conduit à l'écriture de poèmes ceux qui savent capter ses résonances. Tel est le cas de Pierre Jean Jouve qui, comme l'observe Henry Bauchau l'accompagnant dans une promenade sur les bords du lac de Sils Maria, semble "moins regarder le grand paysage devant nous que l'écouter et de ce moment va naître un poème [...]» ${ }^{\mathbf{2 0 4}}$. C'est que le paysage se montre parfois au poète sous les traits d'un poème.

201 Henry Bauchau, L'Écriture à l'écoute, Arles, Actes Sud, 2000, pp. 28-29. Les citations suivantes sont tirées de cette édition.

202 Ibid., p. 43.

203 Henry Bauchau, Les Années difficiles. Journal 1972-1983, Arles, Actes Sud, 2009, p. 368. Les citations suivantes sont tirées de cette édition.

204 L'Écriture à l'écoute, p. 126. 
Ces notes de journal témoignent du fait que la rencontre entre le sujet et le monde environnant est, de même que l'inconscient, le rêve, l'histoire ou le vécu personnel, une circonstance de première importance, dans laquelle peut s'ancrer la genèse d'un écrit.

À partir de ces propos de l'écrivain, nous allons examiner comment la fréquentation de la montagne et son appréhension quotidienne pendant les années 19511975 concourent en partie à l'élaboration de cet imaginaire du minéral si prégnant dans l'œuvre.

Nous considérerons le paysage au sens que lui donne Michel Collot ${ }^{\mathbf{2 0 5}}$, à savoir l'image du pays tel qu'il est perçu par un observateur ou représenté par un artiste. À l'opposé de l'espace, qui désigne selon le critique une étendue distincte de la res cogitans homogène, universelle et mathématisable, et à la différence du lieu, circonscrit comme le territoire d'une communauté partageant les valeurs d'une même culture, le paysage, pour Michel Collot, est défini par le point de vue d'un sujet et lié à son expérience sensible. Il met donc en jeu un site, un sujet et un langage; il est un lieu qui ne relève pas de l'introspection, mais plutôt d'une projection du sujet lyrique, qui ne peut s'exprimer que dans son rapport conjoint au monde et aux mots. D’où un rapport à double sens : le paysage reflète les émotions du sujet, mais il les suscite aussi.

Les deux journaux de la période suisse parus à ce jour, La Grande Muraille. Journal de La Déchirure (1960-1965) et Les Années difficiles (1972-1983), de même que certaines notes rétrospectives d'autres journaux, révèlent donc la place significative du contact avec le paysage de montagnes à ce moment précis de la vie de Bauchau. Le paysage minéral tant de fois parcouru au fil de longues promenades ou à ski, qu'il soit roche ou massifs entiers, n'est pas une simple toile, mais véritablement une des modalités selon lesquelles l'écrivain appréhende le monde et intériorise ses lois. Ce qui frappe dans l'évocation de ce paysage, c'est sa sensorialité, au sein d'une représentation qui oscille souvent entre réel et imaginaire.

La montagne se manifeste à Henry Bauchau avant tout à travers la vue : "La passion des pierres, le paysage devient presque secondaire. Violets, verts de gloire, vert clair, roses, blancs et veinés de vert, les cailloux me sollicitent et m'enchantent. „206 Cette perception révèle la richesse des couleurs et donne l'impression que l'appréhension du paysage passe par le filtre de la picturalité, précisément au moment où Henry Bauchau s'engage dans cet art et l'enseigne. La couleur a d'ailleurs exercé une grande fascination sur lui, et lors des entretiens avec Albert Palma et Patrice van Eersel $^{\mathbf{2 0 7}}$, Bauchau manifestait sa conviction que la peinture peut rendre la nature, à travers la couleur, avec plus d'objectivité que l'écriture. Or, le besoin de

205 Michel Collot, Paysage et poésie du romantisme à nos jours, Paris, José Corti, 2005.

206 Henry Bauchau, La Grande Muraille. Journal de La Déchirure (1960-1965), Arles, Actes Sud, 2005, coll. Babel, p. 142. Les citations suivantes sont tirées de cette édition.

207 Albert Palma, Le Peuple de la Main, Henry Bauchau sur la route, Alès, Éditions Jean-Paul Bayol, 2007, p. 87. 
prolonger dans l'écriture l'expérience chromatique, ce qui apparaît non seulement dans le passage cité ci-dessus, mais aussi dans certains poèmes écrits pendant la période suisse ("Signes bleus", par exemple, invite le lecteur dans l'univers des "routes jaunes", des "routes rouges", de la "panique blanche» ${ }^{\mathbf{2 0 8}}$ ), montre que la peinture n'était pour lui qu'une des configurations possibles de la couleur.

Mais plus qu'un simple décor perçu visuellement, le paysage minéral de la montagne fonctionne pour lui comme le siège d'une expérience plurisensorielle, que le corps découvre en le parcourant. La pierre est la matière qu'il aime aussi toucher, caresser, modeler: "presque au sommet en remuant les pierres vertes qui abondent, sensation de manier l'or "209, écrit-il. Notons ici la propriété alchimique de la pierre; la richesse naturelle de la matière brute révèle son pouvoir secret à celui qui "éprouve» la matière avec ses mains, avec son corps, qui finit par se sentir aussi puissant, en touchant la pierre, qu'un alchimiste fabriquant de l'or. Jacques Devriend n'évoquait-il pas, lors du colloque de Cerisy, son amitié avec celui qui admirait "le grain et la forme d'une pierre", "cette main qui venait reconnaître et aimer la matière minérale " ${ }^{\mathbf{2 1 0}}$ ? Le bêchage du potager évoqué dans Les Années difficiles parle de l'importance du travail de la matière terrestre, qui a peu à voir avec son utilité, mais représente une autre «vérité de la main» en contact avec la terre, avec la matière de la terre dont je me suis tenu jusqu'à l'an passé si éloigné et je sens que cela me nourrit et me renforce. Il me donne une certaine idée de ce qu'est le travail manuel, de sa dureté, de sa monotonie, mais aussi de sa fécondité. ${ }^{\mathbf{2 1 2}} \mathrm{La}$ relation à la réalité environnante, la "réalité rugueuse à étreindre" dont Rimbaud parle comme d'un regret dans "Adieu ", passe notamment par le corps, et ce rapport redonne poids et sens à une présence corporelle au monde. Un véritable échange de forces s'effectue dans l'approche du minéral et dans sa manipulation; la matière est une ressource qui aide à rétablir l'équilibre physique et psychique, manifestant par là sa dimension thérapeutique dont les écrits de fiction illustrent l'efficacité. Antigone ne se soigne-elle pas avec «un peu de terre sur la blessure» ${ }^{\mathbf{2 1 3}}$ ? Et n'essaie-t-elle pas de guérir son père en appliquant de "la terre argileuse sur sa blessure ${ }^{\mathbf{2 1 4}}$ ?

Si Bauchau se sent tellement attiré par la montagne, c'est parce qu'il a l'impression d'y ressentir des sensations et des émotions qui émanent de la matière même. Le sens olfactif est également sollicité dans cette approche du paysage: "Merveilleuses journées de soleil, la neige est encore présente et les dernières

208 Henry Bauchau, La Pierre sans chagrin. Poèmes du Thoronet, Arles, Actes Sud, 2001 (rééd.), p. 99. Les citations suivantes sont tirées de cette édition.

209 La Grande Muraille, p. 143.

210 Jacques Devriend, "Une rencontre», dans Marc Quaghebeur (dir.), Les Constellations impérieuses d'Henry Bauchau, Bruxelles, AML Éditions/Éditions Labor, 2003, Coll. "Archives du futur", p. 81.

211 L'Écriture à l'écoute, p. 42.

212 Les Années difficiles, p. 141.

213 Henry Bauchau, Antigone, Arles, Actes Sud, 1997, p. 266.

214 Henry Bauchau, Edipe sur la route, Arles, Actes Sud, 1990, p. 25. 
traces de l'hiver, mais surtout vers le soir, on sent poindre le printemps. On sent alors l'odeur de la terre et les montagnes sont entourées, après le coucher du soleil, d'une lumière diffuse et vive qui demande à croître." $2 \mathbf{1 5}$ Bauchau attribue une odeur au monde minéral, alors que cette caractéristique est spécifique aux mondes animal et végétal. Il semble concevoir implicitement une relation indissoluble et osmotique entre les trois règnes, comme s'ils étaient régis par les mêmes lois sensorielles. En effet, les frontières entre les règnes deviennent souvent floues dans ses écrits, où le corps humain devient pierreux, la chambre a "des murs de sapin", les murailles sont "de foin".

La montagne, loin d'augmenter la distance entre l'être et le monde, souvent angoissante, catalyse le dialogue que l'écrivain essaie d'instaurer avec le réel: "Sauf le grand dialogue minéral avec les montagnes, Gstaad est pour moi un lieu où mon être profond n'est que faiblement engagé. „216 Cette phrase met en exergue l'expérience du minéral liée à la montagne: ressentie synesthésiquement dans une richesse sensorielle qui valorise le corps, elle devient un moyen de contact, un support d'émotions et d'enchantement, à tel point que cet échange induit une manière de vivre. Une note du journal du Régiment noir ${ }^{\mathbf{2 1 7}}$ révèle l'adhésion d'Henry Bauchau à la pensée de Giono selon laquelle la vie consisterait à atteindre une plénitude par le truchement des sens, à se faire une "chair spirituelle" de ce que contiennent nos jours. Voilà précisément ce qu'il essaie d'atteindre par l'expérience du paysage montagneux suisse.

Ce paysage n'est donc pas décrit, mais évoqué à travers des images, des rythmes et des sonorités qui retranscrivent à la fois le monde et la présence au monde du sujet. La restitution morcelée du paysage n'est pas sans rapport avec une vision morcelée du Moi. En expliquant la signification de la neige dans La Chine intérieure, le poète affirmait qu'il s'apercevait aussi comme "un petit assemblage de flocons dans l'innombrable assemblée qui enveloppe et recouvre la vallée "218.

Pour Bauchau, la relation au paysage est une expérience des sens prolongée au-delà de la simple perception; sentir signifie «ressentir» dans l'acception donnée par Erwin Straus ${ }^{\mathbf{2 1 9}}$, coïncidant avec un moment "pathique» où le sujet entre dans une communion sympathique ou antipathique avec les choses. Souvent, l'expérience de la contemplation des montagnes ne se limite pas à enregistrer les données sensibles, l'écrivain les organise d'après sa subjectivité et sa sensorialité. La subjectivation du paysage fait que le corps devient co-naturel à ses formes, et que la matière du monde acquiert de son côté la faculté de percevoir. Aussi Henry Bauchau parle-t-il d'«un sentiment de liquidité intérieure qui correspond à celle

215 La Grande muraille, p. 48.

216 Ibid., p. 32.

217 Voir Kerstin Liesegang, "Le journal inédit du Régiment noir», dans Marc Quaghebeur (dir.), Les constellations impérieuses d'Henry Bauchau, op. cit., pp. 171-172.

218 L'Écriture à l'écoute, p. 44.

219 Voir Michel Collot, La Matière-Émotion, Paris, Presses universitaires de France, 2005 [1997], pp. $19-20$. 
du paysage ${ }^{\mathbf{2 2 0}}$. Mélange de réalisme et de lyrisme, la montagne apparaît dans les journaux de Bauchau comme le lieu d'une pureté originelle où une symphonie se laisse entendre à celui qui sait écouter; la beauté du monde s'y dévoile et comble le sujet par ailleurs déchiré par les contraintes de la vie sociale et psychique:

Longue promenade, hier à la fin de l'après-midi, au lac de Lauenen. Un ruisseau dans la forêt descendait sur un lit de roche lisse. C'était un glissement rapide, silencieux, avec quelques accidents de souches ou de pierres. Un bruit de robe longue, un bruit léger sur une robe très fine et protégée comme celle de la face intérieure de la cuisse. Au-dessus de lui le grondement indistinct du torrent, plein de cahots, de ressauts, de franchissements de pierre aux arêtes encore vives. Les deux bruits se mêlaient tout en restant distincts et la musique d'étoffe légère du ruisseau causait sur ce fond confus un très grand plaisir d'oreille. ${ }^{221}$

Dans ce fragment, la sensualité presque érotique des bruits de la montagne, la complémentarité des deux cours du torrent, l'un apaisé et l'autre tumultueux, renvoient d'une certaine manière aux contradictions intérieures du sujet Bauchau et à la complémentarité du yin et du yang du taoïsme chinois. Ce rapprochement entre le paysage minéral suisse et la Chine est repérable également dans d'autres images transposant des paysages intériorisés, dont le lien avec le réel est ténu: «Un pan de montagne apparaissait suspendu au-dessus des sapins. Il était encore en pleine lumière avec quelques arbres, un flanc herbeux sous lequel on devinait la roche et une petite cascade. C'était un morceau de Chine, mais de quelle Chine? Puisque je ne l'ai jamais vue et ne la verrai sans doute jamais. ”22 Ce sont là des paysages rêvés, dont la visite est impossible sinon par une projection imaginaire:

[...] au-dessus de moi la montagne semble suspendue sur ma tête avec un échevellement de roches cernées de sapins et de mélèzes. Cet endroit et la cascade qui suit m'évoquent toujours la Chine. Pourquoi la Chine? [...] La Chine c'est un sommet qui sort des nuées ou c'est un lac avec l'unique barque suspendue entre ciel et terre. ${ }^{223}$

Ce paysage intériorisé et reconfiguré par l'imaginaire, qui appelle l'image de la Chine, équivaut selon Catherine Mayaux $^{\mathbf{2 2 4}}$ à une lecture du monde passant par l'intériorisation de la pensée taoïste chinoise: la présence du cosmos et de la nature, le suspens entre ciel et terre qui laisse place au vide médian, l'alliance de mobilité et d'immobilité s'inscrivent selon la critique dans une conception restituant l'homme au cour des éléments et dans le mouvement des souffles vitaux de l'univers. Cette projection mentale affective renvoie explicitement à la pensée

220 Henry Bauchau, La Déchirure, Bruxelles, Labor, 1986, "Espace Nord», p. 152. Les citations suivantes sont tirées de cette édition.

221 La Grande muraille, p. 78.

222 Idem.

223 Ibid., p. 165

224 Catherine Mayaux, "Habiter la Chine en poète selon Henry Bauchau", dans Catherine Mayaux et Myriam Watthee-Delmotte (dir.), Henry Bauchau. Écrire pour habiter le monde, Saint-Denis, Presses Universitaires de Vincennes, 2009, pp. 261-262. 
orientale, chère à l'auteur, qui postule l'inséparabilité du moi et du cosmos, de l'expérience intérieure et extérieure. Cet échange intime est en Chine à l'origine d'une notion appelée "sentiment-paysage »225; Michel Collot a rappelé que l'échange entre sujet et objet, qui répugne à un esprit occidental, est fondamental dans l'esthétique chinoise. L'adoption de cette conception orientale pourrait expliquer l'évocation de la Chine dans une expérience paysagère qui unit l'atmosphère du paysage à l'état d'âme du sujet. En outre, l'identification qu'établit Henry Bauchau entre Cézanne et la montagne Sainte Victoire confirme l'importance que l'écrivain accorde au rapport sujet-objet, rendu en chinois par un terme qui signifie "hôte - convive", comme Albert Palma l'a souligné226. L'appréhension du paysage minéral de la Suisse par Henry Bauchau relève donc d'une expérience des sens, qui consiste à l'intérioriser et à le transfigurer intérieurement. L'échange qui s'établit entre le sujet et le monde, cette émotion ressentie devant le paysage, poussent le sujet à sortir de soi et à se redécouvrir dans le monde. Le paysage pénètre le sujet et le sujet se prolonge dans le monde, donnant naissance à ce que Merleau-Ponty nommait le "chiasme perceptif " ${ }^{\mathbf{2 2 7}}$, c'est-à-dire l'inversion du dehors et du dedans.

Une longue masse blanche aérienne où les contours des rochers s'étaient effacés dans la lumière diminuée. Une présence lointaine, absolument sereine, et qui me semble intérieure. Je la voyais en face de moi et elle était aussi en moi, non pas comme une partie de mon ego, mais comme un ensemble auquel j'appartiens et que je suis avant toute pensée. ${ }^{\mathbf{2 2 8}}$

On pourrait lire dans ce fragment l'image d'une réalité qui précède la mémoire personnelle, faite de sons et de rythmes, d'un langage originel. D'ailleurs, c'est le rythme du paysage qu'Henry Bauchau perçoit le plus souvent:

Quelque chose dans la plénitude me fait penser à la rumeur du torrent sous ma fenêtre au moment de la fonte des neiges. Dans la profondeur du tumulte, dans l'épaisseur et la richesse de son volume, on entend quelques notes secrètes que j'appelle le bruit du monde. C'est le glissement sur le fond du torrent des pierres et des galets que la force de l'eau déplace. C'est ce mouvement souterrain des surfaces polies les unes sur les autres, ce bruit mat de la pierre entraînée qui se trouve un autre lit, ce sont toutes ces syllabes séparées, brisées et finalement fondues dans la grande phrase anonyme qui font, non pas le râle de maman, mais sa matière et ce profond tissu dans lequel la mort peut coudre. ${ }^{\mathbf{2 9}}$

225 Michel Collot, Paysage et poésie du romantisme à nos jours, op. cit.

226 Henry Bauchau affirmait dans un entretien avec Albert Palma: "Quand [Cézanne] peint la montagne Sainte Victoire, il est la montagne", dans Albert Palma, Le Peuple de la Main, Henry Bauchau sur la route, op. cit., p. 92.

227 Voir Jean-Jacques Wunenburger, Le désert et l'imagination cosmo-poétique, sur http://www.geopoetique.net/archipel_fr/institut/cahiers/col2_jjw.html, consulté le 6 juillet 2009.

228 Les Années difficiles, p. 93.

229 La Déchirure, p. 174. 
Le rapport tendu à la mère, finalement résolu dans la mort, est dit à travers les éléments du paysage; par-delà l'inconscient gardant mémoire de cette relation, ces éléments conservent donc les reflets d'une existence individuelle.

Le paysage se fait rythme intérieur par l'union des perceptions et des sensations venues de l'extérieur qui sont à l'origine de la création littéraire. L'écrivain en témoignera à propos de la pièce de théâtre Gengis Khan: "L'histoire du vent et celle du poème sont profondément liées dans l'écriture. "230 C'est précisément dans ce rythme que réside la genèse des poèmes: "Survient un son, un rythme, une image, une intuition et j'ai soudain le désir, l'espérance d'écrire un poème "231; et la difficulté de l'écriture intervient au moment du passage de la sensation à la verbalisation, à la recherche d'un langage adéquat. Il s'agit pour l'écriture de capter le rythme originel auquel le paysage renvoie souvent, de retrouver l'émotion qui précède le langage et de le transposer en mots avec les moyens adéquats, sans trahir la sensibilité: "L'homme avec la terre dans le poème / fait-il œuvre de musicien?" "232 Ou encore: "Je vis dans ce torrent et j'entends les montagnes / en mots de sourds, la nuit, soupirer leur langage / immobile. Je sens que je pourrais l'apprendre / si j'écoutais assez de temps, et qu'on pourrait / parler à voix de roche et de silence d'herbe. "233 La quête d'une langue par le biais de l'élémentarité du paysage équivaut à un retour à ses sources, à un retour à un "sens plus pur» ${ }^{\mathbf{2 3 4}}$, à même d'exprimer l'intensité de la sensation, qui se fait par l'imitation de la matière, comme le dit La Chine intérieure:

Pendant que peu à peu, par la contemplation de la neige et de l'eau

Tu découvrais sous les paroles

L'imitation de la matière. ${ }^{235}$

Même si l'expérience de la montagne procède des sens, les réflexions que ces transpositions poétiques suscitent l'imaginaire minéral d'Henry Bauchau. Celui-ci apparaît le plus souvent intimement lié à l'eau du torrent. L'affrontement de l'eau et de la pierre se résout dans une alliance qui fait qu'elles s'équilibrent: si la pierre règle l'écoulement de l'eau, celle-ci travaille lentement la matière minérale et modèle ses aspérités. Le minéral subit le travail de l'eau, sans se laisser pourtant emporter par le torrent. La pierre, modelée et polie par l'eau qui lui donne de fins contours sans abîmer sa forme initiale, sera à l'origine d'une analogie récurrente dans l'esthétique bauchalienne, comme le montrent plusieurs passages de ses journaux: "Je travaille depuis plusieurs jours, avec difficulté, au chant d'Edipe sur la

230 L'Écriture à l'écoute, p. 27.

231 Henry Bauchau, "Dépendance amoureuse du poème», dans Heureux les déliants, Bruxelles, Labor, 1995 , p. 19.

232 La Pierre sans chagrin, p. 14.

233 Ibid., p. 15

234 L'Écriture à l'écoute, p. 43.

235 La Pierre sans chagrin, p. 194. 
Sphinx. [...] il s'agit maintenant de polir, sans abîmer ni affadir le texte initial. „236 En outre, la matière verbale est identifiée métaphoriquement aux composantes rocheuse et aquatique du paysage: "la pesanteur et la liquidité des mots» ${ }^{\mathbf{2 3 7}}$.

La fréquence des recours aux images minérales de la montagne semble refléter la tentative de trouver une certaine stabilité et un équilibre destinés à contrecarrer l'état "flottant" qui préoccupait Bauchau à cette époque: "Tout s'en allait dans ces matières devenues molles. Il fallait rebâtir en dur.” ${ }^{238}$ L'appel constant du dur est directement lié à l'idée de durable. La fixité des montagnes répond au désir d'échapper à la dégradation car un des premiers traits du minéral est sa capacité de résister à une destruction trop rapide; il est voué à accomplir le rêve de fixation de l'informe, condensé dans l'image de la vague pétrifiée, devenue célèbre grâce au le chapitre "La Vague» d'Edipe sur la route paru en 1990, mais dont l'écrivain avait déjà l'intuition en mars 1960 lorsqu'il habitait Gstaad: "Certaines montagnes ressemblent vraiment à d'immenses vagues pétrifiées. » ${ }^{239}$ Il avait transposé cette vision dans le poème "Melopée Viking» écrit à cette période: "Quand la rouille du glas et les cris du tocsin s'éteindront / sous l'ortie dans les vagues de pierres / [...] aurons-nous terminé nos funèbres destins?» ${ }^{\mathbf{2 4 0}}$

Ainsi, "la vie plus lente de la pierre», qui s'oppose à "nos durées de courte paille ${ }^{\mathbf{2 4 1}}$, correspond dans l'univers référentiel de Bauchau au mot "cool" que son fils Patrick vient de lui apprendre: "Cool: cette fascination de l'indifférence, cette fascination de la pierre. À rapprocher du sentiment que j'ai eu si souvent de la vie plus lente de la pierre ${ }^{\mathbf{2 4 2}}$. Car le minéral de la montagne, travaillé par le temps autant que l'homme, sait affronter «mieux que nous / l'irrémédiable» et réagit "comme de vieux soldats harassés par la guerre» ${ }^{\mathbf{2 4 3}}$. On connaît la lutte permanente d'Henry Bauchau pour dégager du temps pour écrire, partagé qu'il est entre son activité professionnelle et celle d'écrivain. C'est dans ce sens qu'on pourrait comprendre la vie plus lente du minéral : il s'agirait d'un endiguement, d'une pétrification du temps, d'un désir de "sortie du temps", si nécessaire à la rédaction de l'œuvre, comme le dit une note du journal du Régiment noir ${ }^{\mathbf{2 4 4}}$.

Dans la montagne s'éprouvent aussi la plénitude de l'être, sa force. En commentant un des poèmes de Mao, Bauchau faisait la remarque suivante: "On ne peut séjourner longtemps en haute montagne ou la traverser à pied, comme l'a fait Mao, sans éprouver combien, à l'échelle d'une vie plus vaste et plus puissante que la

236 Henry Bauchau, Jour après jour. Journal 1983-1989, Bruxelles, Labor, 2003, "Babel», p. 258.

237 L'Écriture à l'écoute, p. 29.

238 La Déchirure, p. 55.

239 La Grande Muraille, p. 50.

240 La Pierre sans chagrin, p. 23.

241 Ibid., p. 19.

242 La Grande Muraille, p. 153.

243 La Pierre sans chagrin, p. 15.

244 Voir Kerstin Liesegang, "Le journal inédit du Régiment noir», art. cit., p. 178. 
nôtre, les montagnes ressemblent à un rêve de la matière. Comme l'océan, elles sont pour nous et en nous l'image la plus forte du soulèvement, de la révolution et, en même temps, de la stabilité minérale. „245

Le minéral des montagnes s'apparente pour Henry Bauchau au sentiment de puissance, il possède la force prométhéenne capable de "tenter l'assaut du ciel » ${ }^{\mathbf{2 4 6}}$. Il dit aussi la force d'un caractère comme celui du vieillard aveugle qui parmi les paysans anxieux accompagnant Tchelou T'saï, est l'unique à demeurer "dans l'immobilité minérale» ${ }^{\mathbf{2 4 7}}$. Il renvoie également à la fierté, à l'orgueil qui sont des traits de caractère qu'avoue lui-même l'écrivain: "Étonné chaque jour de constater l'énorme part de vanité de mon caractère, je me moque souvent de moi-même mais la vanité demeure un roc solide.» ${ }^{\mathbf{2 4 8}}$ Après avoir vécu dans le sentiment d'être le second, Bauchau a éprouvé grâce à la direction de l'Institut Montesano l'impression d'une certaine puissance, comme il l'affirmait en 1964 dans La Grande Muraille: "Il y a un plaisir d'être le centre, le directeur de Montesano. » ${ }^{249}$

Pourtant, la stabilité à laquelle Bauchau aspire ne signifie pas réduction à l'inerte, limitation à un état brut, car ce qui est brut lui répugne. Les moments difficiles de sa vie sont parfois rendus en analogie avec la pierre brute, agressive: "le travail lent, pénible et rocailleux sur "La lignée II" ”250 ou bien "Hier soir [1]a respiration étouffée [de maman], un râle qui semblait passer sur des cailloux bruts » ${ }^{\mathbf{5 1}}$. Bauchau rêve d'une nature non pas statique, mais soumise aux lois du devenir, moins rapide que celui de l'homme; d'une transformation non sous la forme de dégradation mais d'affinement.

Pour Bauchau, la montagne est un espace où s'affrontent les contraires. Toute une conception de la vie est révélée par cette représentation: "Pour vivre il fallait pouvoir sinuer sans cesse, comme un ruisseau, entre les blocs "252, acquérir les "mouvements aussi fluides" que ceux de la Sibylle. Ainsi, "la liberté de l'eau contrainte par la pente mais qui danse et rebondit sur le roc ${ }^{\mathbf{2 5 3}}$, que Bauchau a tant de fois admirée, incarne la double faculté de la pierre modelée par un agent extérieur, mais toujours dotée des qualités qui lui sont propres. Cette caractéristique fait écho chez Bauchau à une exigence intime qui suppose d'une part de travailler l'inerte et d'autre part d'endiguer le flux, de pétrifier l'informe, comme l'a mis en évidence Jacques Poirier ${ }^{254}$.

245 Henry Bauchau, Essai sur la vie de Mao Zedong, Paris, Flammarion, 1982, pp. 419-420.

246 La Pierre sans chagrin, p. 50.

247 Henry Bauchau, Gengis Khan, Arles, Actes Sud, coll. Papiers, 1989, p. 95.

248 La grande muraille, p. 214.

249 Ibid., p. 350.

250 Ibid., p. 138.

251 Ibid., p. 105.

252 La Déchirure, p. 116.

253 La Grande Muraille, p. 70.

254 Voir à ce propos l'article de Jacques Poirier, "Le rocher et la vague: morceler, dissoudre, représenter", dans Marc Quaghebeur (dir.), Les Constellations impérieuses d'Henry Bauchau, op. cit., pp. 
C'est en cela que réside la grandeur d'un caractère comme celui du personnage de Peyrol, rencontré au fil des lectures: "Fini hier soir Le Frère-de-la-côte. Le personnage de Peyrol est irrécusable. Il y a en lui quelque chose qui rappelle la beauté d'une lourde pierre longtemps roulée et polie par le torrent. „255 En revanche, l'inaptitude à se laisser modeler et affiner engendre des caractères bruts comme celui de Gengis Khan: «je ne suis qu'un roc. Une pierre frappée par la foudre et qui va dévalant les montagnes. Je ne vis pas. Je n'ai jamais vécu» ${ }^{256}$.

La vie dans les Alpes suisses reste un épisode important pour Henry Bauchau; elle a marqué un tournant dans son processus de reconfiguration identitaire, à travers l'ouverture vers le monde et l'enracinement dans la matière. L'auteur même en avait conscience le 23 juillet 1961: "Il y a exactement dix ans que je suis à Gstaad. [...] Il est vrai que c'est durant cette période que, après m'être déraciné, j'ai repris racine dans la matière et par l'esprit. „257 Il y a eu la révélation de la matérialité qui lui est constitutive, de sa condition de petite partie appartenant à un Tout: "Malgré tous les problèmes vécus entre 1951 et 1975, j’ai retrouvé le côté fastueux de l'existence vivant à la montagne. [...] J'ai vécu dans un grand théâtre naturel, sans trop le savoir, j’ai été enveloppé, imprégné par lui.»²5

Le paysage minéral suisse est donc pour Henry Bauchau bien plus que l'expression d'un territoire géographique: il est un pays mystérieux, invitant à l'exploration de ce qui se cache sous sa surface. Plus qu'un décor quotidien, ce paysage s'apparente pour l'écrivain à une vraie expérience intérieure; plutôt qu'un point d'ancrage, il est le moment d'un parcours.

\section{Corina Dambean}

Université Petru Maior de Târgu Mures et Université de Cergy-Pontoise 